\title{
Urinary Biomarkers of Tubular Damage Are Associated with Mortality but Not Cardiovascular Risk among Systolic Blood Pressure Intervention Trial Participants with Chronic Kidney Disease
}

\author{
Vasantha K. Jotwani ${ }^{a, b}$ Alexandra K. Lee Michelle M. Estrella $^{a, b}$ Ronit Katz ${ }^{c}$ \\ Pranav S. Garimellad Rakesh Malhotra d, e Dena E. Rifkin ${ }^{d, e}$ Walter Ambrosius ${ }^{f}$ \\ Barry I. Freedman ${ }^{f} \quad$ Alfred K. Cheung ${ }^{g}$ Kalani L. Raphaelg Paul Drawz ${ }^{\text {h }}$ \\ Javier A. Neyrai Suzanne Oparil ${ }^{j} \quad$ Henry Punzik ${ }^{k}$ Michael G. Shlipak ${ }^{a, b}$ \\ Joachim H. Ix d, e for the SPRINT Research Group

\begin{abstract}
${ }^{a}$ Department of Medicine, San Francisco VA Medical Health Care System, San Francisco, CA, USA; ${ }^{b}$ Kidney Health Research Collaborative, San Francisco VA Medical Center and University of California, San Francisco, CA, USA; ${ }^{C}$ Kidney Research Institute, University of Washington, Seattle, WA, USA; ${ }^{d}$ Department of Medicine, University of California, San Diego, CA, USA; ${ }^{e}$ Veterans Affairs San Diego Healthcare System, San Diego, CA, USA; ${ }^{\text {Department of }}$ Medicine, Wake Forest School of Medicine, Winston-Salem, NC, USA; ${ }^{9}$ Department of Medicine, University of Utah, Salt Lake City, UT, USA; ${ }^{\text {h}}$ Department of Medicine, University of Minnesota, Minneapolis, MN, USA; 'Department of Medicine, University of Kentucky, Lexington, KY, USA; ${ }^{j}$ Department of Medicine, University of Alabama at Birmingham, Birmingham, AL, USA; ${ }^{k}$ Punzi Medical Center, Trinity Hypertension Research Institute,
\end{abstract} \\ Carollton, TX, USA
}

\section{Keywords}

Urinary biomarkers · Kidney injury · Interleukin-18 ·

Chitinase-3-like protein-1

\begin{abstract}
Background: Kidney tubulointerstitial fibrosis on biopsy is a strong predictor of chronic kidney disease (CKD) progression, and CKD is associated with elevated risk of cardiovascular disease (CVD). Tubular health is poorly quantified by traditional kidney function measures, including estimated glomerular filtration rate (eGFR) and albuminuria. We hypothesized that urinary biomarkers of tubular injury, inflammation, and repair would be associated with higher risk of CVD and mortality in persons with CKD. Methods: We measured urinary concentra-
\end{abstract}

(c) 2019 S. Karger AG, Basel

E-Mail karger@karger.com www.karger.com/ajn tions of interleukin-18 (IL-18), kidney injury molecule-1, neutrophil gelatinase-associated lipocalin, monocyte chemoattractant protein-1, and chitinase-3-like protein-1 (YKL-40) at baseline among 2,377 participants of the Systolic Blood Pressure Intervention Trial who had an eGFR $<60 \mathrm{~mL} / \mathrm{min} / 1.73 \mathrm{~m}^{2}$. We used Cox proportional hazards models to evaluate biomarker associations with CVD events and all-cause mortality. Results: At baseline, the mean age of participants was $72 \pm$ 9 years, and eGFR was $48 \pm 11 \mathrm{~mL} / \mathrm{min} / 1.73 \mathrm{~m}^{2}$. Over a median follow-up of 3.8 years, 305 CVD events (3.6\% per year) and 233 all-cause deaths ( $2.6 \%$ per year) occurred. After multivariable adjustment including eGFR, albuminuria, and urinary creati-

M.G.S. and J.H.I. co-senior authors.
Vasantha Jotwani, MD 
nine, none of the biomarkers showed statistically significant associations with CVD risk. Urinary IL-18 (hazard ratio [HR] per 2 -fold higher value, 1.14; 95\% Cl 1.01-1.29) and YKL-40 (HR per 2 -fold higher value, $1.08 ; 95 \% \mathrm{Cl} 1.02-1.14)$ concentrations were each incrementally associated with higher mortality risk. Associations were similar when stratified by randomized blood pressure arm. Conclusions: Among hypertensive trial participants with CKD, higher urinary IL-18 and YKL-40 were associated with higher risk of mortality, but not CVD.

(C) 2019 S. Karger AG, Basel

\section{Introduction}

Chronic kidney disease (CKD), when assessed by decreased estimated glomerular filtration rate (eGFR) or higher albuminuria, is associated with increased risk of cardiovascular disease (CVD) [1-3]. Advanced tubular damage, characterized by tubular atrophy and interstitial fibrosis on kidney biopsy, is a strong predictor of CKD progression $[4,5]$, but tubular damage is inadequately captured by eGFR and albuminuria, which primarily mark glomerular function and injury [6, 7]. Several urinary biomarkers have been investigated for detection of kidney tubular injury, inflammation, and repair in persons with and without CKD. Whether or not these novel dimensions of kidney tubular health prognosticate CKD complications, including CVD and mortality, is uncertain.

Interleukin-18 (IL-18), kidney injury molecule-1 (KIM-1), and neutrophil gelatinase-associated lipocalin (NGAL) have been studied extensively as markers of tubular injury, with urine levels rising several fold in response to ischemic or inflammatory acute kidney injury (AKI) [814]. Monocyte chemoattractant protein-1 (MCP-1) is a chemokine that attracts macrophages to the site of injury [15-17], whereas chitinase-3-like protein-1 (YKL-40) functions as a mediator of the reparative response to tubular injury $[18,19]$. Some studies have demonstrated that urinary markers of tubular damage are associated with higher cardiovascular and mortality risks [17, 20-22], whereas other studies found no significant associations $[23,24]$. Few of the existing studies included large numbers of participants with CKD who may have more extensive tubule damage and higher risk for CVD and death. In addition, no prior study, to our knowledge, has evaluated urinary YKL-40, a marker of renal tubular repair, as a predictor of cardiovascular complications in CKD.

The objective of this study was to evaluate the associations of urinary markers of tubular injury (IL-18, KIM-1, and NGAL) and tubular inflammation and repair (MCP-
1 and YKL-40) with the risks of CVD and mortality among participants of the Systolic Blood Pressure Intervention Trial (SPRINT) who had CKD at baseline. We hypothesized that these urinary markers would be associated with higher risk of CVD events and mortality, independent of eGFR and albuminuria.

\section{Methods}

Study Design and Participants

SPRINT was an open-label clinical trial that randomized persons with systolic blood pressure (SBP) $>130 \mathrm{~mm} \mathrm{Hg}$ and elevated risk for CVD events to an SBP target of $<120 \mathrm{~mm} \mathrm{Hg}$ ("intensive") vs. $<140 \mathrm{~mm} \mathrm{Hg}$ ("standard") [25]. Trial results were published previously [26, 27]. Participants were recruited from 102 centers in the United States and Puerto Rico and were required to meet the following inclusion criteria: age $\geq 50$ years, SBP $130-180 \mathrm{~mm} \mathrm{Hg}$, and increased risk for CVD events (defined by: prior clinical or subclinical CVD other than stroke, 10 -year risk of CVD of $15 \%$ or greater based on the Framingham risk score [28], CKD defined as eGFR $20-59 \mathrm{~mL} / \mathrm{min} / 1.73 \mathrm{~m}^{2}$, or age $\geq 75$ years). Major exclusion criteria included diabetes mellitus, proteinuria $>1 \mathrm{~g} /$ day, polycystic kidney disease, prior stroke or transient ischemic attack, symptomatic heart failure, or a left ventricular ejection fraction $<35 \%$. A total of 9,361 participants were enrolled between November 2010 and March 2013. Efforts to increase recruitment of participants with CKD resulted in the enrollment of 2,646 (28\%) participants with $\mathrm{eGFR}<60 \mathrm{~mL} / \mathrm{min} / 1.73 \mathrm{~m}^{2}$ at baseline, based on the Modification of Diet in Renal Disease equation. SPRINT randomly assigned participants in a 1:1 ratio to the intensive or standard blood pressure arm. The antihypertensive regimens were adjusted to maintain SBP according to the randomized treatment target. Participants attended visits monthly for the first 3 months and every 3 months thereafter; clinical data were obtained at serial visits. Venous blood and urine specimens were processed immediately, shipped overnight on dry ice, and stored at $-80^{\circ} \mathrm{C}$ at a central laboratory. All participants provided written informed consent, and Institutional Review Boards of all participating institutions approved the study. The present study was conducted in accordance with the Declaration of Helsinki and was also approved by the committees on human research at the University of California, San Francisco, and the San Francisco and San Diego Veterans Affairs Health Care Systems.

We measured urinary biomarkers at the baseline visit among persons with $\mathrm{CKD}$, defined by the CKD-EPI equation that combines serum creatinine and cystatin C [29]. Among the 2,514 SPRINT participants who had an eGFR $<60 \mathrm{~mL} / \mathrm{min} / 1.73 \mathrm{~m}^{2}$ by this equation, 86 participants were excluded due to unavailable urine specimens or invalid urinary biomarker measurements and 51 participants were excluded due to missing covariate data, resulting in a final analytic sample of 2,377 participants.

\section{Predictors}

Urinary IL-18, KIM-1, NGAL, MCP-1, and YKL-40 were measured at the Laboratory for Clinical Biochemistry Research at the University of Vermont. All urine specimens were in continuous storage at $-80^{\circ} \mathrm{C}$ until biomarker measurement without prior thaw. Laboratory personnel performing the biomarker assays were 
blinded to clinical information about the participants. Biomarker measurements were performed by multiplex assays (Meso Scale Diagnostics, Rockville, MD, USA). Inter-assay coefficients of variation for the urine measurements were IL-18, 4.9-13.7\%; KIM-1, 6.1-13.0\%; NGAL, 12.9-16.2\%; MCP-1, 7.1-12.0\%; and YKL-40, $6.5-11.1 \%$. Biomarkers were measured in duplicate and averaged to increase precision. Urine creatinine and albumin were measured by an enzymatic procedure (Roche, Indianapolis, IN, USA) and by a nephelometric method (Siemens, Tarrytown, NY, USA), respectively. Inter-assay coefficients of variation for urine creatinine and albumin measurements were $1.5-4.3 \%$ and $2.2-6.9 \%$, respectively. Samples with biomarker values below the limit of detection were assigned a value equivalent to the lower limit of detection divided by the square root of 2 [30].

\section{Outcomes}

The outcomes of this study included CVD events, the primary outcome of the SPRINT trial, and all-cause mortality. CVD events were defined as a composite of nonfatal myocardial infarction, acute coronary syndrome, nonfatal acute decompensated heart failure, nonfatal stroke, and death from CVD causes. Ascertainment and adjudication of these outcomes have been described previously [25].

\section{Statistical Analysis}

Baseline characteristics of participants were compared across quartiles of each biomarker. Spearman coefficients were used to evaluate correlations among the urinary biomarkers, urine albumin, urine creatinine, and eGFR calculated by the CKD-EPI equation that combines serum creatinine and cystatin $C$ [29]. We used Cox proportional hazards models to evaluate associations of each urinary biomarker with CVD events and all-cause mortality in separate models. Participants were censored at death or last available study follow-up. Covariates for multivariable models were selected based on biological plausibility and included: age, sex, race (non-Hispanic white, non-Hispanic black, other), intervention arm, urine creatinine, eGFR (CKD-EPI equation for creatinine and cystatin C), urine albumin, smoking status (current, former, never), history of CVD or heart failure, number of antihypertensive medications, statin use, SBP, diastolic blood pressure, body mass index, high-density lipoprotein, total cholesterol, and triglycerides. Baseline values were used for all covariates.

The optimal method for correcting biomarker concentrations for differences in urinary tonicity has not been established. Because urine creatinine is influenced by muscle mass and health status and may therefore influence the ratio of biomarkers to creatinine [31], our primary analyses evaluated biomarker concentrations that adjusted for urine creatinine concentration in the multivariable models. In secondary analyses, the biomarkers were indexed to urine creatinine as biomarker/creatinine ratios. Due to their right-skewed distributions, biomarker levels (with or without indexing to creatinine) were analyzed as log base 2-transformed continuous variables, with results presented per 2-fold higher value. We also evaluated biomarker levels by quartile, with participants in the lowest quartile comprising the reference group. Finally, we tested for interactions by randomization arm using a likelihood ratio test. The proportional hazards assumption was satisfied for all models by visual inspection of the log negative and log survival curves and by the Schoenfeld residuals. All analyses were conducted using Stata/ MP version 15.1 (StataCorp LCC, College Station, TX, USA).

\section{Results}

Baseline Characteristics of SPRINT CKD Participants

Among the 2,377 participants included in this study, 1,222 participants were randomized to the intensive treatment arm and 1,155 participants were randomized to the standard treatment arm. The mean age was 72 years, and $40 \%$ of participants were women. Non-Hispanic blacks comprised $26 \%$ of participants and $25 \%$ of participants had a history of CVD or heart failure. The median eGFR was $48 \mathrm{~mL} / \mathrm{min} / 1.73 \mathrm{~m}^{2}$ (interquartile range 39, 55), and the median ACR was $14 \mathrm{mg} / \mathrm{g}$ (interquartile range 7, 46). Compared to participants in the lowest quartile of urinary YKL-40 concentration, those in the highest quartile were older, more likely to be female or non-Hispanic black, and had lower prevalence of CVD or heart failure (Table 1). Compared to participants in the lowest quartile of urinary IL-18 concentration, those in the highest quartile were younger, more likely to be female or non-Hispanic black, and had higher body mass index (online suppl. Table 1; for all online suppl. material, see www.karger.com/ doi/10.1159/000499531). When we compared participants with and without subsequent CVD events (online suppl. Table 2) or mortality events (online suppl. Table 3) during follow-up, each type of event occurred more commonly in participants characterized by older age, male gender, prevalent CVD or heart failure at baseline, smoking history, lower eGFR, higher albuminuria, and randomization to the standard BP arm of the trial.

\section{Correlations of Urinary Biomarkers and eGFR}

Urinary concentrations of IL-18, KIM-1, NGAL, MCP-1, and YKL-40 showed moderately strong intercorrelations (online suppl. Table 4; $r=0.4-0.7, p<0.05$ ), and each marker was positively correlated with urine albumin $(r=0.3-0.4 ; p<0.05)$ and urine creatinine $(r=0.3-0.7$; $p<0.05)$ concentrations. The urinary markers showed little correlation with eGFR $(|r|<0.1$ for all 5 markers). After indexing to urine creatinine, correlations between the biomarkers were attenuated substantially $(r=0.1-0.5$; $p<0.05)$.

\section{Associations of Urinary Biomarkers with CVD Events and All-Cause Mortality}

Over a median follow-up of 3.8 years, there were 305 CVD events (3.6\% per year) and 233 all-cause deaths (2.6\% per year). In demographic-adjusted models, higher urine IL-18, NGAL, MCP-1, and YKL-40 concentrations were each associated with higher risk for CVD events (Table 2). However, after additional adjustment for eGFR, 
Table 1. Baseline characteristics of SPRINT participants with CKD by quartile of urinary YKL-40 $(n=2,377)$

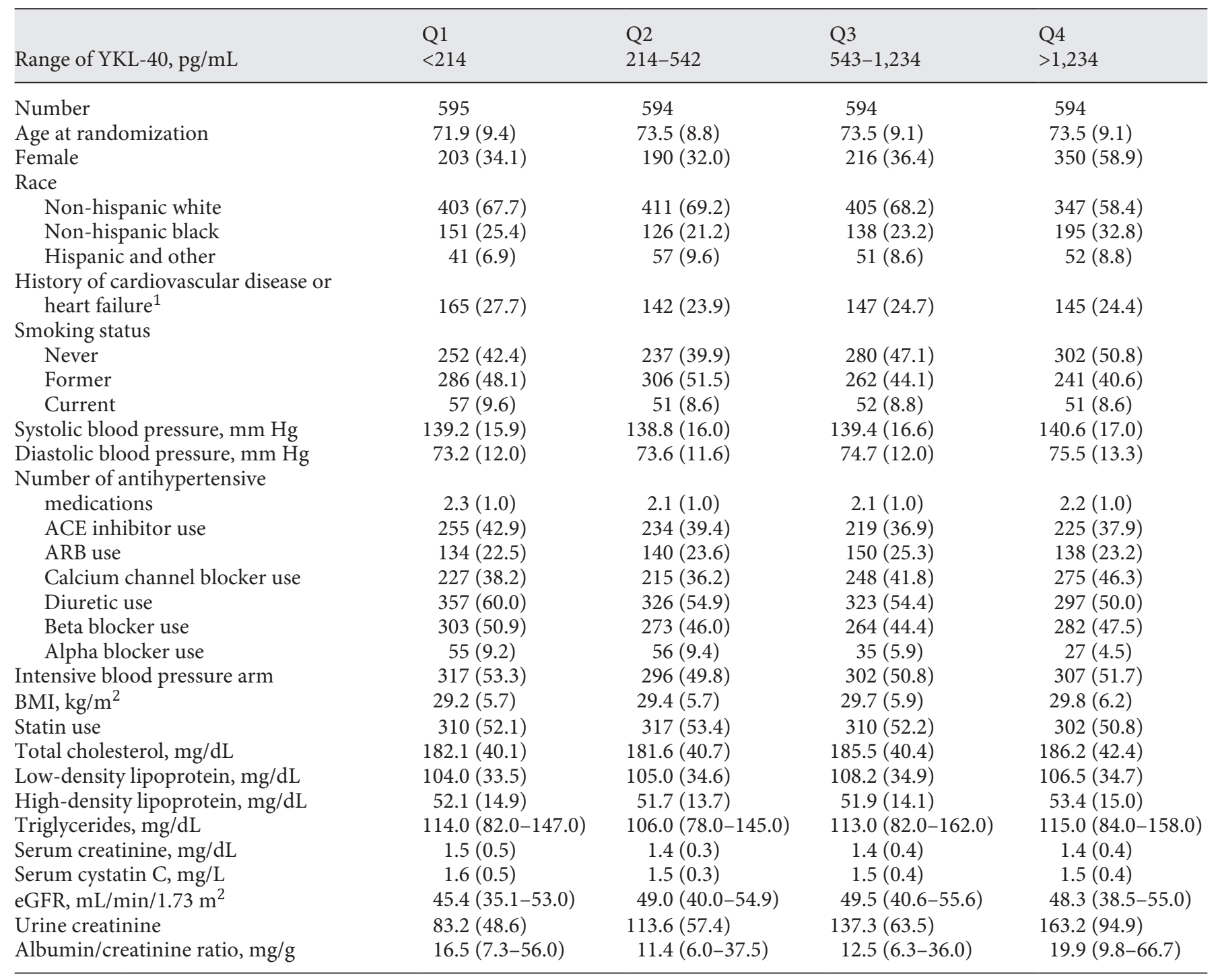

Data are presented as median (IQR), mean $\pm \mathrm{SD}$, or numbers (percent).

${ }^{1}$ Includes any one of the following: Previous myocardial infarction, percutaneous coronary intervention, coronary artery bypass surgery, carotid endarterectomy, carotid stenting, peripheral artery disease with revascularization, acute coronary syndrome with or without resting ECG change, ECG change on graded exercise test, or positive cardiac imaging study, at least a $50 \%$ diameter stenosis of a coronary, carotid, or lower extremity artery, abdominal aortic aneurysm $\geq 5 \mathrm{~cm}$ with or without repair, or self-reported heart failure.

ACE inhibitor, angiotensin-converting enzyme inhibitor; ARB, angiotensin receptor blocker; eGFR, estimated glomerular filtration rate by combined CKD-EPI equation for serum creatinine and cystatin C; YKL-40, chitinase-3-like protein-1. BMI, body mass index; SPRINT, Systolic Blood Pressure Intervention Trial; IQR, interquartile range.

albuminuria, and traditional CVD risk factors, all associations were attenuated and none remained statistically significant. When the biomarkers were analyzed as quartiles (Fig. 1), participants in the highest quartiles of IL-18 (hazard ratio [HR] 1.43; 95\% CI 0.95-2.14) and YKL-40 (HR 1.41; 95\% CI 0.99-2.00) had higher risk of CVD events than those in the lowest quartiles, but the associations did not reach statistical significance. When the biomarkers were indexed to urine creatinine (online suppl. Table 5), participants in the highest versus lowest quartile of YKL-40/Cr had higher risk of CVD events (HR 1.41; 95\% CI 1.01-1.99), but urinary YKL-40/Cr was not asso- 
Table 2. Associations of urinary biomarkers with CVD events and all-cause mortality among SPRINT participants with CKD $(n=2,377)$

\begin{tabular}{|c|c|c|}
\hline & $\begin{array}{l}\text { CVD events } \\
\text { HR }(95 \% \text { CI })\end{array}$ & $\begin{array}{l}\text { All-cause mortality } \\
\text { HR }(95 \% \mathrm{CI})\end{array}$ \\
\hline \multicolumn{3}{|l|}{ Urine IL-18 } \\
\hline Demographic-adjusted $^{1}$ & $1.15(1.04-1.27)$ & $1.22(1.08-1.37)$ \\
\hline Multivariable-adjusted $^{2}$ & $1.06(0.96-1.18)$ & $1.14(1.01-1.29)$ \\
\hline \multicolumn{3}{|l|}{ Urine KIM-1 } \\
\hline Demographic-adjusted $^{1}$ & $1.06(0.98-1.16)$ & $1.15(1.03-1.28)$ \\
\hline Multivariable-adjusted $^{2}$ & $0.93(0.86-1.02)$ & $0.96(0.86-1.06)$ \\
\hline \multicolumn{3}{|l|}{ Urine NGAL } \\
\hline Demographic-adjusted $^{1}$ & $1.09(1.02-1.17)$ & $1.11(1.02-1.20)$ \\
\hline Multivariable-adjusted $^{2}$ & $1.03(0.96-1.11)$ & $1.03(0.95-1.12)$ \\
\hline \multicolumn{3}{|l|}{ Urine MCP-1 } \\
\hline Demographic-adjusted $^{1}$ & $1.13(1.02-1.24)$ & $1.13(1.01-1.27)$ \\
\hline Multivariable-adjusted $^{2}$ & $1.04(0.94-1.15)$ & $1.03(0.91-1.17)$ \\
\hline \multicolumn{3}{|l|}{ Urine YKL-40 } \\
\hline Demographic-adjusted $^{1}$ & $1.07(1.01-1.13)$ & $1.11(1.05-1.19)$ \\
\hline Multivariable-adjusted $^{2}$ & $1.04(0.99-1.10)$ & $1.08(1.02-1.14)$ \\
\hline
\end{tabular}

\footnotetext{
${ }^{1}$ Adjusted for age, sex, race, intervention arm, and urine creatinine.

${ }^{2}$ Adjusted for age, sex, race, intervention arm, urine creatinine, baseline eGFR by combined CKD-EPI equation for serum creatinine and cystatin $\mathrm{C}$, urine albumin, smoking status, history of cardiovascular disease or heart failure, number of antihypertensive medications, statin use, systolic blood pressure, diastolic blood pressure, body mass index, high-density lipoprotein, total cholesterol, and triglycerides.

HRs per doubling of baseline urinary biomarker concentration.

CVD, cardiovascular disease; IL-18, interleukin-18; KIM-1, kidney injury molecule-1; MCP-1, monocyte chemoattractant protein-1; NGAL, neutrophil gelatinase-associated lipocalin; YKL-40, chitinase-3-like protein-1; SPRINT, Systolic Blood Pressure Intervention Trial; CKD, chronic kidney disease; HR, hazard ratio.
}

ciated with CVD events when analyzed as a continuous variable. Urinary IL-18/Cr, KIM-1/Cr, NGAL/Cr, and $\mathrm{MCP}-1 / \mathrm{Cr}$ were not associated with CVD events in fully adjusted models.

In demographic-adjusted models, higher urinary IL18, KIM-1, NGAL, MCP-1, and YKL-40 concentrations were each associated with higher mortality risk (Table 2). After additional adjustment for baseline eGFR, albuminuria, and traditional CVD risk factors, higher urinary IL18 and YKL-40 remained significantly associated with higher mortality risk. By contrast, the associations of urinary KIM-1, NGAL and MCP-1 with mortality were substantially attenuated and were no longer statistically significant in fully adjusted models. When the biomarkers were analyzed as quartiles (Fig. 2), participants in the highest vs. lowest quartiles of urinary YKL-40 had significantly higher mortality risk in multivariable-adjusted models (HR 1.70; 95\% CI 1.12-2.57). Findings were similar when the biomarkers were indexed to urine creatinine (online suppl. Table 6), with higher urinary IL-18/Cr (HR 1.20 per 2 -fold higher value; 95\% CI 1.05-1.36) and YKL-
40/Cr (HR 1.08 per 2-fold higher value; 95\% CI 1.02-1.14) each incrementally associated with higher mortality risk in multivariable models. Relative to participants in the lowest quartiles, those in the highest quartiles of urinary IL-18/Cr and YKL-40/Cr had higher CVD risk by 1.71fold (95\% CI 1.15-2.53) and 1.92-fold (95\% CI 1.27-2.92), respectively. Urinary KIM-1/Cr, NGAL/Cr, and MCP-1/ Cr showed no statistically significant associations with allcause mortality in fully adjusted models.

There were no statistically significant interactions by intervention arm for the CVD outcome $(p>0.1$ for all biomarkers) or for all-cause mortality ( $p>0.3$ for all biomarkers).

\section{Discussion}

Despite growing recognition of the adverse effects of kidney tubular damage on CKD progression, the prognostic significance of tubular damage for cardiovascular complications and mortality has been uncertain. We hy- 
Fig. 1. Multivariable-adjusted associations of urinary biomarkers with CVD events among SPRINT participants with CKD ( $n=2,377)$. HRs (95\% CI) displayed per quartile of baseline biomarker concentrations. Multivariable models adjust for age, sex, race, intervention arm, urine creatinine, baseline eGFR by combined CKD-EPI equation for serum creatinine and cystatin C, urine albumin, smoking status, history of cardiovascular disease or heart failure, number of antihypertensive medications, statin use, systolic blood pressure, diastolic blood pressure, body mass index, high-density lipoprotein, total cholesterol, and triglycerides. IL-18, interleukin-18; KIM-1, kidney injury molecule-1; MCP-1, monocyte chemoattractant protein-1; NGAL, neutrophil gelatinase-associated lipocalin; YKL-40, chitinase-3-like protein-1.

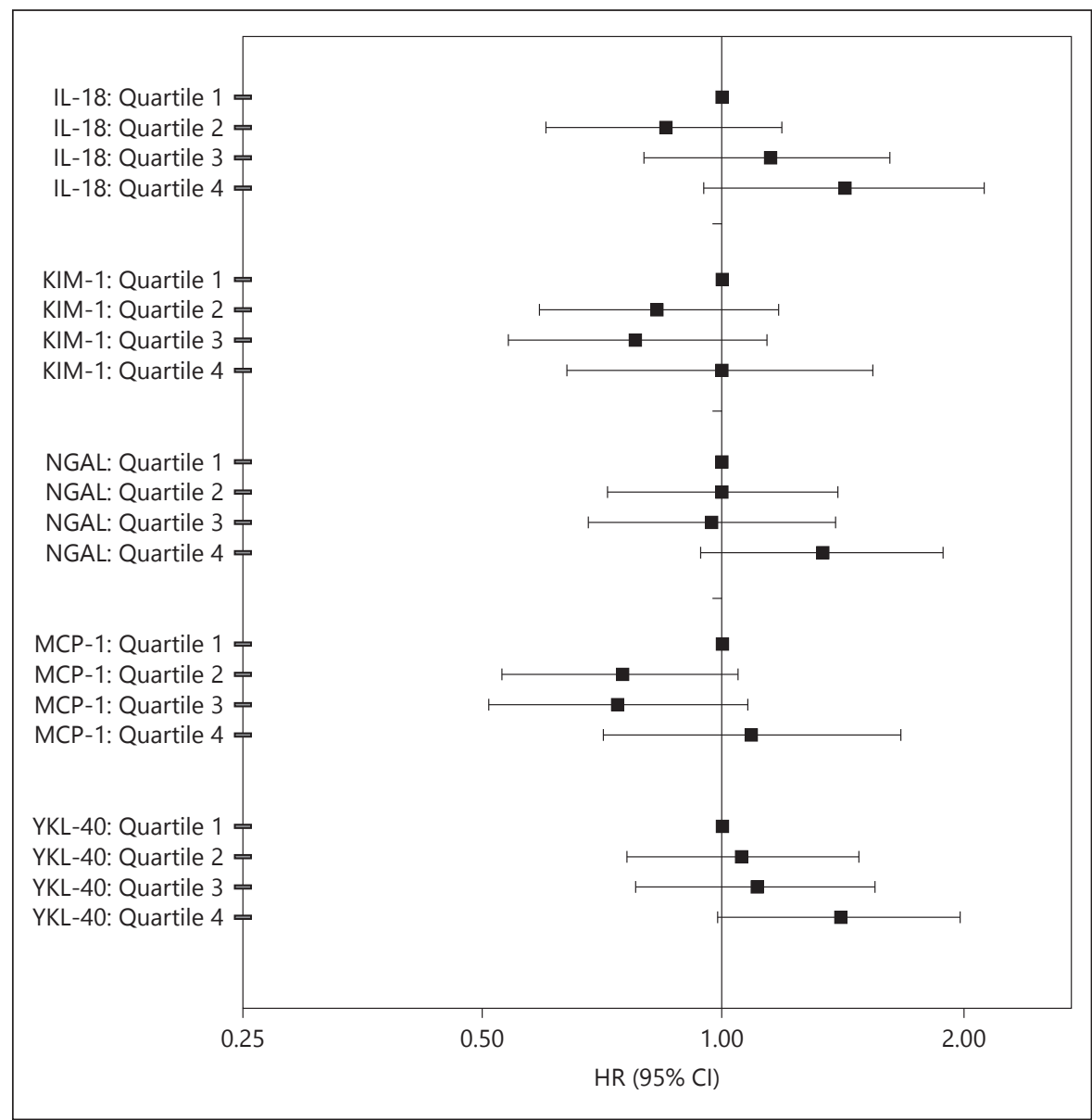

As kidney diagnostics evolve to encompass multiple dimensions of kidney health, there has been considerable interest in determining whether markers that evaluate the health of kidney tubules can provide prognostic information beyond the established measures of glomerular health, eGFR, and albuminuria. Kidney tubular health is necessary for numerous essential functions, including solute and water reabsorption, acid-base homeostasis, hormone production, and mineral metabolism [32]. Due to high metabolic demands, the kidney tubules are vulnerable to injury from ischemic, inflammatory, or toxic insults, and incomplete repair following injury can lead to the development of CKD [32-36]. However, tubular injury, inflammation, and repair processes are poorly captured by eGFR and albuminuria. Our findings indicate that urinary biomarkers of kidney tubular injury and repair have independent associations with increased mortality risk in persons with CKD, but associations with CVD were weaker and generally absent. Further studies are needed to understand whether the associations with mortality represent a causal link or a shared pathogenesis. 
Fig. 2. Multivariable-adjusted associations of urinary biomarkers with all-cause mortality among SPRINT participants with CKD $(n=2,377)$. HRs (95\% CI) displayed per quartile of baseline biomarker concentrations. Multivariable models adjust for age, sex, race, intervention arm, urine creatinine, baseline eGFR by combined CKDEPI equation for serum creatinine and cystatin C, urine albumin, smoking status, history of cardiovascular disease or heart failure, number of antihypertensive medications, statin use, systolic blood pressure, diastolic blood pressure, body mass index, high-density lipoprotein, total cholesterol, and triglycerides. IL-18, interleukin-18; KIM-1, kidney injury molecule-1; MCP-1, monocyte chemoattractant protein-1; NGAL, neutrophil gelatinase-associated lipocalin; YKL-40, chitinase-3-like protein-1.

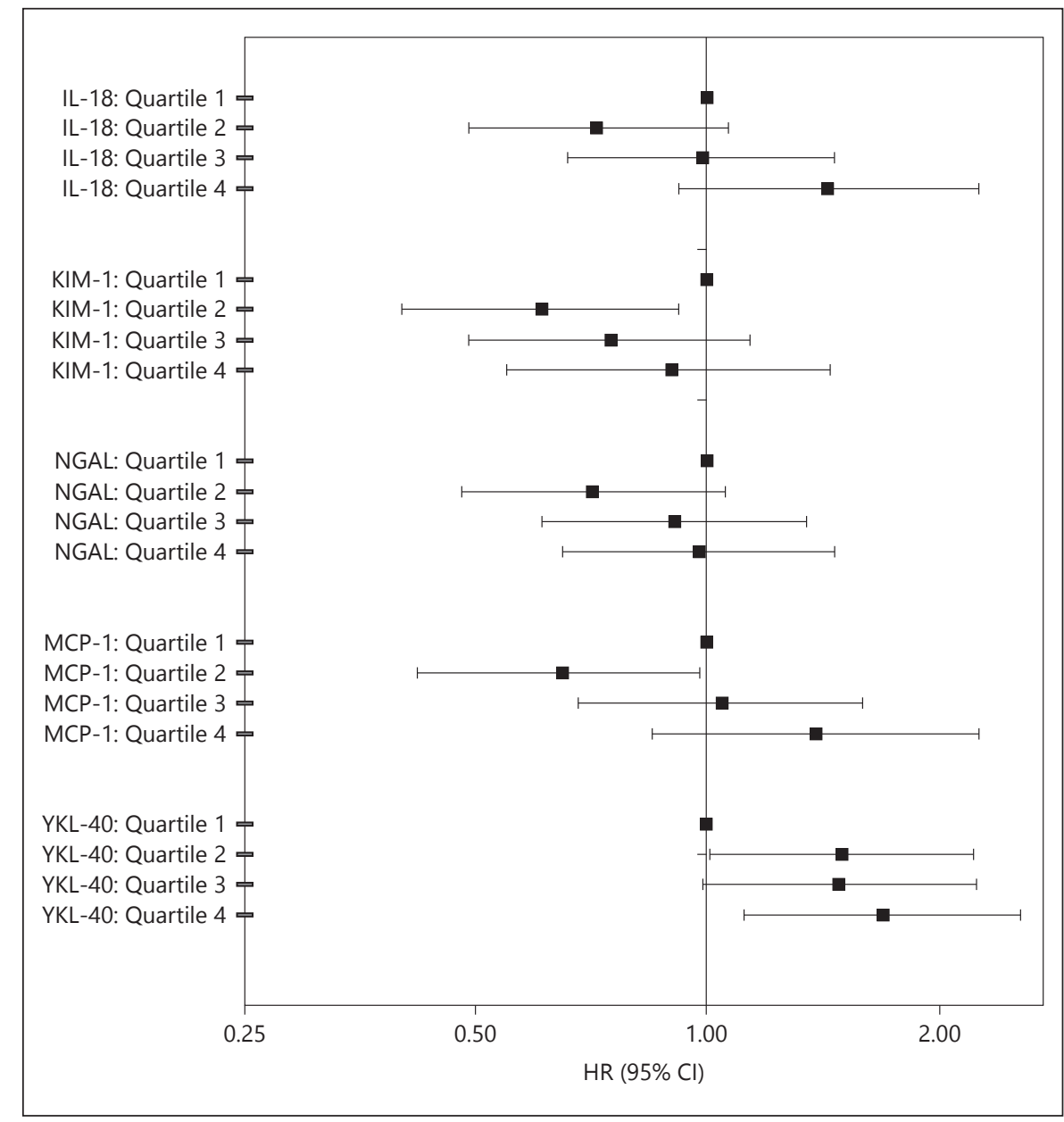

Previous studies have examined urinary markers of tubular injury and clinical outcomes in ambulatory settings. In the Health, Aging and Body Composition cohort of elders, who predominantly had preserved kidney function, higher urinary KIM-1 and NGAL were associated with higher risks of heart failure and CVD, respectively, and both were associated with increased mortality risk over a median follow-up of 12.4 years [21, 23, 37]. Higher urinary IL-18 levels were also associated with increased mortality risk in a cohort of HIV-infected individuals with preserved kidney function [22]. Additionally, in the Chronic Renal Insufficiency Cohort (CRIC), a cohort of persons with CKD, higher urinary KIM-1 was associated with heart failure and ischemic atherosclerotic events and both urinary KIM-1 and NGAL were associated with higher risks of death over a median follow-up of 6.5 years [20]. In our study of SPRINT participants with CKD, higher urinary IL-18 and YKL-40 were each associated with higher mortality risk, but in contrast to the CRIC and Health Aging and Body Composition studies, none of the markers were associated with CVD risk. These discordant findings may be explained by the relatively short duration of follow-up in SPRINT or by differences in the study population, which was substantially older in SPRINT than in CRIC and excluded diabetes. Taken together with the existing literature, our findings suggest that more extensive kidney tubular injury is a risk factor for mortality, although the individual biomarker associations differ across cohorts. In future investigations, it will be necessary to develop methods for integrating information from intercorrelated biomarkers, which may reflect overlapping pathophysiology within the kidney.

Because biomarker concentrations are influenced by the tonicity of urine specimens, some prior studies have indexed biomarker concentrations to urine creatinine, similar to the albumin/creatinine ratio that is used in clinical practice. However, this approach may overestimate biomarker associations with adverse outcomes, due to the impact of worse overall health status and lower muscle mass on lower urine creatinine concentra- 
tions, which can increase the biomarker/creatinine ratio. Our primary analyses, therefore, evaluated urine creatinine-adjusted biomarker concentrations, and we evaluated creatinine-indexed biomarkers in secondary analyses to enable comparison with previous studies. Overall, we observed qualitatively similar effect sizes across the biomarkers for each outcome. However, in creatinine-indexed analyses, we found that the highest quartile of YKL-40/Cr was significantly associated with higher risk for CVD. Although the preferred analytic method remains uncertain, our findings suggest that the interpretation of future urinary biomarker studies must take into consideration the approach for creatinine standardization.

To our knowledge, the association of urinary YKL-40 with increased risk of mortality among persons with CKD has not been reported previously. Translational studies identified YKL-40 as a urinary marker of sepsis-induced AKI [18] and as a critical component of the renal repair response following ischemia-reperfusion injury [19]. Among 249 hospitalized patients with AKI, higher urinary YKL-40 concentrations were associated with a composite outcome of AKI progression and/or death [38]. By contrast, the direction of association in a subsequent study of 1,301 kidney donors was different. This study found that higher donor urinary YKL-40 concentrations were associated with reduced risk of delayed graft function, suggesting that YKL-40 may positively impact the response to acute tubular injury [39]. In the ambulatory setting, a nested case-control study within the Action to Control Cardiovascular Risk in Diabetes trial found no association between urinary YKL-40 and kidney function decline during follow-up [40]. Our study builds upon the relatively sparse literature on urinary YKL-40 by demonstrating novel associations with mortality risk among persons with CKD.

There are several limitations of this study. First, because we evaluated a cohort of predominantly older nondiabetic individuals with CKD, our results may not be generalizable to younger populations or persons with diabetes or earlier stages of kidney damage. Second, although we did not observe statistically significant differences by randomization arm, our study was not designed to evaluate whether urinary biomarker levels will inform specific blood pressure treatment strategies. Third, urinary biomarkers were measured at baseline only, and we did not have access to serum levels. Finally, although we adjusted for potential confounders in our multivariable models, we cannot exclude the possibility of residual confounding.

Kidney Tubular Damage and Risk of CVD and Mortality
In this study of hypertensive individuals with CKD, higher urinary IL-18 and YKL-40 were associated with higher mortality risk, independent of eGFR and albuminuria, but not with CVD risk. Further studies are needed to validate these findings and to investigate the mechanisms underlying these associations.

\section{Acknowledgments and Funding Sources}

This ancillary study was supported by the National Institute of Diabetes and Digestive and Kidney Diseases (R01DK098234 for MGS/JHI, K24DK110427 for JHI, and K23DK109868 for VJ) and the American Heart Association (14EIA18560026 for JHI). The authors thank the participants and staff members of the SPRINT, which was funded with Federal funds from the National Institutes of Health, including the National Heart, Lung, and Blood Institute, the National Institute of Diabetes and Digestive and Kidney Diseases, the National Institute on Aging, and the National Institute of Neurological Disorders and Stroke, under Contract Numbers HHSN268200900040C,HHSN268200900046C,HHSN268200900047C, HHSN268200900048C, HHSN268200900049C, and Inter-Agency Agreement Number A-HL-13-002-001. It was also supported in part with resources and use of facilities through the Department of Veterans Affairs. The SPRINT investigators acknowledge the contribution of study medications (azilsartan and azilsartan combined with chlorthalidone) from Takeda Pharmaceuticals International, Inc. All components of the SPRINT study protocol were designed and implemented by the investigators. The investigative team collected, analyzed, and interpreted the data. All aspects of manuscript writing and revision were carried out by the coauthors. The content is solely the responsibility of the authors and does not necessarily represent the official views of the National Institutes of Health, the US Department of Veterans Affairs, or the United States Government. For a full list of contributors to SPRINT, please see the supplementary acknowledgement list: https://www.sprinttrial.org/public/ dspScience.cfm.

We also acknowledge the support from the following CTSAs funded by NCATS: CWRU: UL1TR000439, OSU: UL1RR025755, U Penn: UL1RR024134\& UL1TR000003, Boston: UL1RR025771, Stanford: UL1TR000093, Tufts: UL1RR025752, UL1TR000073 \& UL1TR001064, University of Illinois: UL1TR000050, University of Pittsburgh: UL1TR000005, UT Southwestern: 9U54TR000017-06, University of Utah: UL1TR000105-05, Vanderbilt University: UL1 TR000445, George Washington University: UL1TR000075, University of CA, Davis: UL1 TR000002, University of Florida: UL1 TR000064, University of Michigan: UL1TR000433, Tulane University: P30GM103337 COBRE Award NIGMS, Wake Forest University: UL1TR001420.

\section{Disclosure Statement}

M.G.S.: has worked as a consultant for the University of Washington and has equity in TAI Diagnostics and Cricket Health, Inc. J.H.I.: holds an investigator initiated research grant from Baxter International Inc. The results presented in this paper have not been published previously in whole or part, except in abstract form. 


\section{References}

1 Sarnak MJ, Levey AS, Schoolwerth AC, Coresh J, Culleton B, Hamm LL, et al.; American Heart Association Councils on Kidney in Cardiovascular Disease, High Blood Pressure Research, Clinical Cardiology, and Epidemiology and Prevention. Kidney disease as a risk factor for development of cardiovascular disease: a statement from the American Heart Association Councils on Kidney in Cardiovascular Disease, High Blood Pressure Research, Clinical Cardiology, and Epidemiology and Prevention. Circulation. 2003 Oct;108(17):215469.

2 Matsushita K, van der Velde M, Astor BC, Woodward M, Levey AS, de Jong PE, et al.; Chronic Kidney Disease Prognosis Consortium. Association of estimated glomerular filtration rate and albuminuria with all-cause and cardiovascular mortality in general population cohorts: a collaborative meta-analysis. Lancet. 2010 Jun;375(9731):2073-81.

3 Waheed S, Matsushita K, Sang Y, Hoogeveen $\mathrm{R}$, Ballantyne C, Coresh J, et al. Combined association of albuminuria and cystatin Cbased estimated GFR with mortality, coronary heart disease, and heart failure outcomes: the Atherosclerosis Risk in Communities (ARIC) Study. Am J Kidney Dis. 2012 Aug;60(2):207-16.

4 Howie AJ, Ferreira MA, Adu D. Prognostic value of simple measurement of chronic damage in renal biopsy specimens. Nephrol Dial Transplant. 2001 Jun;16(6):1163-9.

5 Nath KA. Tubulointerstitial changes as a major determinant in the progression of renal damage. Am J Kidney Dis. 1992 Jul;20(1):117.

6 Kassirer JP. Clinical evaluation of kidney function-glomerular function. N Engl J Med. 1971 Aug;285(7):385-9.

7 Rule AD, Amer H, Cornell LD, Taler SJ, Cosio FG, Kremers WK, et al. The association between age and nephrosclerosis on renal biopsy among healthy adults. Ann Intern Med. 2010 May;152(9):561-7.

8 Parikh CR, Abraham E, Ancukiewicz M, Edelstein CL. Urine IL-18 is an early diagnostic marker for acute kidney injury and predicts mortality in the intensive care unit. J Am Soc Nephrol. 2005 Oct;16(10):3046-52.

9 Parikh CR, Jani A, Melnikov VY, Faubel S, Edelstein CL. Urinary interleukin-18 is a marker of human acute tubular necrosis. Am J Kidney Dis. 2004 Mar;43(3):405-14.

10 Parikh CR, Mishra J, Thiessen-Philbrook H, Dursun B, Ma Q, Kelly C, et al. Urinary IL-18 is an early predictive biomarker of acute kidney injury after cardiac surgery. Kidney Int. 2006 Jul;70(1):199-203.

11 Ichimura T, Hung CC, Yang SA, Stevens JL, Bonventre JV. Kidney injury molecule-1: a tissue and urinary biomarker for nephrotoxicant-induced renal injury. Am J Physiol Renal Physiol. 2004 Mar;286(3):F552-63.
12 Han WK, Bailly V, Abichandani R, Thadhani $\mathrm{R}$, Bonventre JV. Kidney Injury Molecule-1 (KIM-1): a novel biomarker for human renal proximal tubule injury. Kidney Int. 2002 Jul; 62(1):237-44.

13 Mishra J, Dent C, Tarabishi R, Mitsnefes MM, Ma Q, Kelly C, et al. Neutrophil gelatinaseassociated lipocalin (NGAL) as a biomarker for acute renal injury after cardiac surgery. Lancet. 2005 Apr;365(9466):1231-8.

14 Parikh CR, Jani A, Mishra J, Ma Q, Kelly C, Barasch J, et al. Urine NGAL and IL-18 are predictive biomarkers for delayed graft function following kidney transplantation. Am J Transplant. 2006 Jul;6(7):1639-45.

15 Munshi R, Johnson A, Siew ED, Ikizler TA, Ware LB, Wurfel MM, et al. MCP-1 gene activation marks acute kidney injury. J Am Soc Nephrol. 2011 Jan;22(1):165-75.

16 Tam FW, Sanders JS, George A, Hammad T, Miller C, Dougan T, et al. Urinary monocyte chemoattractant protein-1 (MCP-1) is a marker of active renal vasculitis. Nephrol Dial Transplant. 2004 Nov;19(11):2761-8.

17 Park M, Katz R, Shlipak MG, Weiner D, Tracy R, Jotwani V, et al. Urinary Markers of Fibrosis and Risk of Cardiovascular Events and Death in Kidney Transplant Recipients: the FAVORIT Trial. Am J Transplant. 2017 Oct; 17(10):2640-9.

18 Maddens B, Ghesquiere B, Vanholder R, et al. Chitinase-like proteins are candidate biomarkers for sepsis-induced acute kidney injury. Mol Cell Proteomics. 2012 Jun; 11(6):M111.013094.

19 Schmidt IM, Hall IE, Kale S, Lee S, He CH, Lee Y, et al. Chitinase-like protein Brp-39/ YKL-40 modulates the renal response to ischemic injury and predicts delayed allograft function. J Am Soc Nephrol. 2013 Feb;24(2): 309-19.

20 Park M, Hsu CY, Go AS, Feldman HI, Xie D, Zhang X, et al.; Chronic Renal Insufficiency Cohort (CRIC) Study Investigators; CKD Biomarkers Consortium. Urine Kidney Injury Biomarkers and Risks of Cardiovascular Disease Events and All-Cause Death: the CRIC Study. Clin J Am Soc Nephrol. 2017 May;12(5):761-71.

21 Jotwani V, Katz R, Ix JH, Gutiérrez OM, Bennett M, Parikh CR, et al. Urinary biomarkers of kidney tubular damage and risk of cardiovascular disease and mortality in elders. Am J Kidney Dis. 2018 Aug;72(2):205-13.

22 Peralta C, Scherzer R, Grunfeld C, et al. Urinary biomarkers of kidney injury are associated with all-cause mortality in the Women's Interagency HIV Study (WIHS). HIV Med. 2014 May;15(5):291-300.

23 Sarnak MJ, Katz R, Newman A, Harris T, Peralta CA, Devarajan P, et al.; Health ABC Study. Association of urinary injury biomarkers with mortality and cardiovascular events. J Am Soc Nephrol. 2014 Jul;25(7): 1545-53.
24 Parikh CR, Puthumana J, Shlipak MG, Koyner JL, Thiessen-Philbrook H, McArthur E, et al. Relationship of Kidney Injury Biomarkers with Long-Term Cardiovascular Outcomes after Cardiac Surgery. J Am Soc Nephrol. 2017 Dec;28(12):3699-707.

25 Ambrosius WT, Sink KM, Foy CG, Berlowitz DR, Cheung AK, Cushman WC, et al.; SPRINT Study Research Group. The design and rationale of a multicenter clinical trial comparing two strategies for control of systolic blood pressure: the Systolic Blood Pressure Intervention Trial (SPRINT). Clin Trials. 2014 Oct;11(5):532-46.

26 Wright JT Jr, Williamson JD, Whelton PK, Snyder JK, Sink KM, Rocco MV, et al.; SPRINT Research Group. A Randomized Trial of Intensive versus Standard Blood-Pressure Control. N Engl J Med. 2015 Nov; 373(22):2103-16.

27 Cheung AK, Rahman M, Reboussin DM, Craven TE, Greene T, Kimmel PL, et al.; SPRINT Research Group. Effects of Intensive BP Control in CKD. J Am Soc Nephrol. 2017 Sep; 28(9):2812-23.

28 D'Agostino RB Sr, Vasan RS, Pencina MJ, Wolf PA, Cobain M, Massaro JM, et al. General cardiovascular risk profile for use in primary care: the Framingham Heart Study. Circulation. 2008 Feb;117(6):743-53.

29 Levey AS, Stevens LA, Schmid CH, Zhang YL, Castro AF 3rd, Feldman HI, et al.; CKD-EPI (Chronic Kidney Disease Epidemiology Collaboration). A new equation to estimate glomerular filtration rate. Ann Intern Med. 2009 May;150(9):604-12.

30 Hornung RW, Reed LD. Estimation of Average Concentration in the Presence of Nondetectable Values. Appl Occup Environ Hyg. 1990;5(1):46-51.

$31 \mathrm{Ix} \mathrm{JH}$, de Boer IH, Wassel CL, Criqui MH, Shlipak MG, Whooley MA. Urinary creatinine excretion rate and mortality in persons with coronary artery disease: the Heart and Soul Study. Circulation. 2010 Mar;121(11): 1295-303.

32 Brenner BM, Rector FC. Brenner \& Rector's the kidney. 8th ed. Philadelphia: Saunders Elsevier; 2008.

33 Hsu CY, Chertow GM, McCulloch CE, Fan D, Ordoñez JD, Go AS. Nonrecovery of kidney function and death after acute on chronic renal failure. Clin J Am Soc Nephrol. 2009 May; 4(5):891-8.

34 Lo LJ, Go AS, Chertow GM, McCulloch CE, Fan D, Ordoñez JD, et al. Dialysis-requiring acute renal failure increases the risk of progressive chronic kidney disease. Kidney Int. 2009 Oct; 76(8):893-9.

35 Ishani A, Nelson D, Clothier B, Schult T, Nugent S, Greer N, et al. The magnitude of acute serum creatinine increase after cardiac surgery and the risk of chronic kidney disease, progression of kidney disease, and death. Arch Intern Med. 2011 Feb;171(3):226-33. 
36 Coca SG, Yusuf B, Shlipak MG, Garg AX, Parikh CR. Long-term risk of mortality and other adverse outcomes after acute kidney injury: a systematic review and meta-analysis. Am J Kidney Dis. 2009 Jun;53(6):96173.

37 Driver TH, Katz R, Ix JH, Magnani JW, Peralta CA, Parikh CR, et al.; Health ABC Study. Urinary kidney injury molecule 1 (KIM-1) and interleukin 18 (IL-18) as risk markers for heart failure in older adults: the Health, Aging, and Body Composition (Health ABC) Study. Am J Kidney Dis. 2014 Jul;64(1):49-56.

38 Hall IE, Stern EP, Cantley LG, Elias JA, Parikh CR. Urine YKL-40 is associated with progressive acute kidney injury or death in hospitalized patients. BMC Nephrol. 2014 Aug;15(1): 133.

39 Puthumana J, Hall IE, Reese PP, Schröppel B, Weng FL, Thiessen-Philbrook $\mathrm{H}$, et al.
YKL-40 Associates with Renal Recovery in Deceased Donor Kidney Transplantation. J Am Soc Nephrol. 2017 Feb;28(2):66170.

40 Nadkarni GN, Rao V, Ismail-Beigi F, Fonseca VA, Shah SV, Simonson MS, et al. Association of Urinary Biomarkers of Inflammation, Injury, and Fibrosis with Renal Function Decline: the ACCORD Trial. Clin J Am Soc Nephrol. 2016 Aug;11(8):1343-52. 